\title{
Arborescences
}

Revue d'études françaises

\section{« Hydrargyre » de Maurice de Fleury ou l'imagination de la matière}

\section{Lola Kheyar Stibler}

Numéro 8, décembre 2018

La Seine littéraire au XIX ${ }^{\mathrm{e}}$ siècle

URI : https://id.erudit.org/iderudit/1055886ar

DOI : https://doi.org/10.7202/1055886ar

Aller au sommaire du numéro

\section{Éditeur(s)}

Département d'études françaises, Université de Toronto

ISSN

1925-5357 (numérique)

Découvrir la revue

Citer cet article

Kheyar Stibler, L. (2018). « Hydrargyre » de Maurice de Fleury ou l'imagination de la matière. Arborescences, (8), 102-113. https://doi.org/10.7202/1055886ar
Résumé de l'article

La nouvelle « Hydrargyre » (1887) de Maurice de Fleury raconte la mort de deux amants, intoxiqués par la Seine métamorphosée en fleuve de mercure. Ce " poème en prose décadent » interroge les modalités d'une esthétisation de la matière et celles de sa portée symbolique, tout en soulevant la question de l'esthétisation de la prose elle-même. En effet, la Seine épaisse et dense y évoque par la négative la métaphore de l'eau pure et transparente couramment assignée à décrire la pureté du grand style français. Sont ainsi pensées ensemble « l'imagination matérielle » de l'eau (Bachelard), la description fantasmagorique du paysage et les composantes stylistiques du texte. Nous interrogeons la façon dont le topos polymorphe et ambivalent de la Seine permet à l'auteur de promouvoir une esthétique symptomatique des enjeux littéraires et stylistiques de la fin du XIX ${ }^{\mathrm{e}}$ siècle.
Tous droits réservés @ Département d'études françaises, Université de Toronto, 2019 document est protégé par la loi sur le droit d’auteur. L’utilisation des services d'Érudit (y compris la reproduction) est assujettie à sa politique d'utilisation que vous pouvez consulter en ligne.

https://apropos.erudit.org/fr/usagers/politique-dutilisation/ 


\section{SOMMAIRE}

1 Nicolas Gauthier, Université de Waterloo

Sébastien Roldan, chercheur indépendant

Introduction

6 Janine Gallant, Université de Moncton

La Seine de Mérimée et ses ressorts dramatiques

17 Silvia Baroni, Université de Bologne

Au seuil de l'enfer: la Seine dans La Comédie humaine

33 Nicolas Gauthier, Université de Waterloo

La Seine du crime et les petits métiers parisiens

46 Sébastien Roldan, chercheur indépendant

Où est la Seine dans les Tableaux parisiens de Baudelaire?

64 Valérie Narayana, Université Mount Allison

Géocritique du fleuve dans les mémoires de la Commune de Louise Michel

80 Peter Vantine, Saint Michael's College

Au bord de la Seine avec les Goncourt

102 Lola Kheyar Stibler, Université Sorbonne Nouvelle

«Hydrargyre» de Maurice de Fleury ou l'imagination de la matière 


\title{
«Hydrargyre» de Maurice de Fleury ou l'imagination de la matière
}

\author{
Lola Kheyar Stibler, Université Sorbonne Nouvelle
}

\section{Résumé}

La nouvelle "Hydrargyre» (1887) de Maurice de Fleury raconte la mort de deux amants, intoxiqués par la Seine métamorphosée en fleuve de mercure. Ce "poème en prose décadent» interroge les modalités d'une esthétisation de la matière et celles de sa portée symbolique, tout en soulevant la question de l'esthétisation de la prose elle-même. En effet, la Seine épaisse et dense y évoque par la négative la métaphore de l'eau pure et transparente couramment assignée à décrire la pureté du grand style français. Sont ainsi pensées ensemble «l'imagination matérielle» de l'eau (Bachelard), la description fantasmagorique du paysage et les composantes stylistiques du texte. Nous interrogeons la façon dont le topos polymorphe et ambivalent de la Seine permet à l'auteur de promouvoir une esthétique symptomatique des enjeux littéraires et stylistiques de la fin du $\mathrm{XIX}^{\mathrm{e}}$ siècle.

En mai 1887 paraît dans La Revue indépendante une nouvelle de dix pages, "Hydrargyre», écrite par un jeune médecin de 27 ans nommé Maurice de Fleury (1860-1931 ${ }^{1}$ ). Celui-ci entretient des relations étroites avec Émile Zola et Joris-Karl Huysmans, avec lesquels il correspond dans les années 18801890. Fervent admirateur de l'auteur des Rougon-Macquart, Fleury conseille Zola pour Le Docteur Pascal (1893) et confie son admiration dans un article du Figaro, en $1896^{2}$. Très "à la mode» parmi les "intellectuels» (selon le mot de Victor Segalen ${ }^{3}$ ), le jeune médecin figure également dans la liste des auteurs symbolistes - aux côtés de Paul Adam, Henri de Régnier et Gustave Kahn - dans un essai d'André Barre, en 1911 (93).

Baptisé de l'autre nom savant du mercure, le récit fantastique «Hydrargyre» raconte la mort de deux amants intoxiqués par une Seine brusquement métamorphosée en fleuve vif-argent. Au début de la nouvelle, l'amant furieux poursuit dans la capitale sa maitresse nue, en pleine nuit; tous deux se précipitent dans le fleuve qui se met à rouler de lourds flots de mercure, resplendissants comme «un métal en fusion» (Fleury, «Hydrargyre»: 264). Jusqu’à une aube sinistre et apocalyptique, les deux amants pétrifiés sont emportés par une eau dense et épaisse, semblable à une "coulée de lave» (264), avant de se retrouver totalement seuls, en rase campagne. La contrée désertique inspire à l'homme une «hiératique incantation» (268) qui rend hommage au mercure salvateur dont le charme mortifère est

1. Membre de l'Académie de Médecine (1909) et spécialisé en psychiatrie, Fleury est notamment l'auteur d'une Introduction à la médecine de l'esprit (1898), qui propose une synthèse de ses observations et méthodes scientifiques; ancien élève de Charcot, Fleury expose dans ses développements quelques-uns des résultats relatifs à la psychologie expérimentale, comme ceux liés à la pratique de l'hypnose et, à l'instar du Docteur Toulouse (Enquête Médicopsychologique sur les rapports de la supériorité intellectuelle avec la névropathie, 1896), il s'intéresse aux relations entre génie et névrose.

2. Sur la correspondance entre Huysmans et Fleury, voir Robert Baldick (1958: 152-153). L'écrivain décrit au médecin ses voyages et son séjour à Hambourg (en août 1888). Sur les relations amicales entre Zola et Fleury, voir Victor Segalen (1902: 68). Voir également l’article que Fleury fait paraître en novembre 1896 dans Le Figaro.

3. Voir Annie Joly-Segalen et Gabriel Germain (1964: 430-431). 
si puissant que les deux amants syphilitiques s'imaginent guérir et, dans un dernier assaut du désir, s'unissent en roulant, toujours emportés par la Seine. Enfin, parvenus à un immense lac de mercure, ils voient jaillir une «aiguille monolithique géante» (271) dont les inscriptions hermétiques signalent l'entrée dans un mystérieux royaume. L'intoxication au mercure commence à gagner les amants dont la mort est finalement suggérée par l'arrivée silencieuse d'un bateau noir, chargé d'explosifs.

Il faut attendre la seconde version de ces quelques pages «fiévreuses et étranges» (Stead 2000: 56), augmentée de deux sections, pour que cette nouvelle soit explicitement rattachée au rêve du personnage principal, l'amant ${ }^{4}$. Le nouveau titre de la nouvelle, «Poème en prose décadent» (1891), supplante le titre laconique de 1887 en signalant une appartenance générique et renvoie, sinon à une "école» décadente, du moins à un imaginaire d'époque marqué par le mysticisme (Nordau 2010:79-100), les visions fantasmagoriques prenant pour objet la matière et ses états intermédiaires (Pierrot 1977: 257292). La publication de la nouvelle dans La Revue indépendante, à côté des productions de Joris-Karl Huysmans, de Jules Laforgue et d'Édouard Dujardin, tout comme la dédicace de la première version au peintre Odilon Redon permettent de situer l'œuvre dans la mouvance symboliste et décadente ${ }^{5}$ et au cœur d'un renouveau mystique qui culmine dans les années 1890 (autour du spiritisme, du magnétisme et de l'occultisme ${ }^{6}$ ). C'est en ce sens qu'on peut interpréter la persistance de l'épigraphe baudelairienne de la première à la deuxième version d' "Hydrargyre»; la référence au "Rêve parisien ", «[...] terrible paysage/Que jamais œil humain ne vit» (Baudelaire, "Rêve parisien»: 10427), est un hommage au père spirituel de la décadence autant qu'un marqueur de filiation intertextuelle. En partageant la fascination des auteurs décadents pour les matières liquides et mobiles ${ }^{8}$, en livrant une description érudite et scientifique des symptômes syphilitiques, Fleury cherche clairement à se situer dans le champ vaste et complexe des esthétiques de la fin du siècle. Les traits de style de son récit lui-même sont aussi bien savants et techniques que lyriques et peuvent relever de ce que certains détracteurs appellent une langue «byzantine», une "langue nouvelle» (Claveau 1886), opposée aux principes et

4. C'est dans le recueil de nouvelles de Fleury intitulé Amours de savants (1891) que la deuxième version d' «Hydrargyre» est publiée; la première version de 1887 est complétée par deux séquences: un jeune étudiant en chimie est interrompu, par une ancienne maîtresse, en pleine préparation d'une leçon d'agrégation traitant «Du mercure et de ses applications en thérapeutique» (section I); il se réveille avec elle le lendemain matin (section III). Le texte de 1887, qui constitue la section II, devient le rêve du jeune savant, la veille d'une épreuve importante. Pour une étude comparative plus détaillée des deux versions de 1887 et 1891, voir Stead (2000).

5. Il n'entre pas dans notre propos de discuter de la pertinence d'une distinction entre ces courants esthétiques. Voir Pierrot (1977: 16) et Stead (2004: 17-30).

6. Le point culminant de la vogue occultiste se remarque notamment par la parution d'une autre revue, LInitiation (1888-1912), qui entend regrouper toutes les tendances des mouvements hermétiques: élucubrations de médecine psychosomatiques de Papus, doctrine gnostique, extraits des œuvres poétiques de Stanislas de Guaïta, comptes rendus d'expériences alchimiques, etc. Les collaborateurs de la revue s'entendent sur l'affirmation d'un principe commun: le refus du matérialisme positiviste. Les deux plus importantes revues littéraires des années 1890, Le Mercure de France et La Revue blanche, informent aussi leurs lecteurs de l'actualité du mouvement occultiste, dont l'alchimie était l'un des thèmes privilégiés.

7. Nous citons la version originale, publiée dans la Revue contemporaine en 1860, dont les variantes sont signalées par Claude Pichois dans l'édition de la Pléiade (1975: 1042); la version définitive parue dans les Tableaux parisiens varie: "Tel que jamais mortel n'en vit» (Baudelaire, «Rêve parisien»: 101 ; nous soulignons).

8. Le roman Bruges-la-morte (1892) de Georges Rodenbach en témoigne notamment. Voir à ce sujet le numéro 21 de la revue Dix-neuf (Emery et Forrest 2017), consacré à la représentation des matières liquides, aux effets de transparence et d'opacité dans les récits décadents. 
aux valeurs de la langue classique faisant l'objet d'un nouveau crédit à la fin du siècle, aussi bien chez la critique conservatrice que chez Maupassant et Zola.

En fusionnant l'eau et le mercure, le liquide et le métal, la nouvelle de Fleury interroge les modalités d'une esthétisation de la matière et celles de sa portée symbolique, tout en posant la question de l'esthétisation de la prose elle-même. Seront ainsi pensées ensemble «l'imagination matérielle» de l'eau (Bachelard 1942: 12), la description fantasmagorique du paysage et les composantes stylistiques du texte, afin d'analyser le traitement singulier que la nouvelle réserve à la Seine. Nous verrons d'abord que le fleuve nocturne d' «Hydrargyre», épais et dense, opaque et lourd, évoque par la négative une métaphore récurrente dans le discours critique, celle de «l'eau pure» et transparente, servant à décrire la pureté du grand style français. Le discours critique des années 1880-1910 et la prose poétique de Fleury partagent en effet leur vocabulaire et s'échangent leurs réseaux métaphoriques. Nous observerons ensuite que l'opacité et la compacité de la matière liquide dans la nouvelle de Fleury débouchent sur une vision fantasmagorique de la Seine, appelant en définitive des considérations métapoétiques et réflexives. Les analyses syntaxiques et lexicologiques permettront dans un dernier temps d'observer la façon dont se répondent les caractéristiques stylistiques de la nouvelle et les motifs qu'elle déploie, notamment celui du délitement. Ainsi, nous questionnerons la façon dont le topos polymorphe et ambivalent de la Seine permet à l'auteur de promouvoir une esthétique singulière, symptomatique des enjeux littéraires et stylistiques de la fin du $\mathrm{XIX}^{\mathrm{e}}$ siècle.

\section{La langue déliquescente: métaphores stylistiques}

La métaphore de l'eau et du flux connaît une fortune critique notable dans les deux dernières décennies $\mathrm{du} \mathrm{XIX}^{\mathrm{e}}$ siècle. Le recours à cette analogie permet de comprendre les lieux communs qui s'instaurent dans le discours critique de l'époque en mettant en avant l'ensemble des représentations liées à un «imaginaire» de la langue, à ses valeurs et à ses normes (Philippe 2013: 211). L'analogie de la langue française et de «l'eau pure» est en effet récurrente chez les promoteurs de la clarté et de la transparence en matière de style, et ce, au moins depuis le XVII siècle: "Le beau langage ressemble à une eau pure et nette qui n'a point de goût", écrit le grammairien Dominique Bouhours (1673: 116). Dans les années 1880 , l'idée d'une transparence du langage constitue un véritable mythe dans les arts d'écrire, par nature prescriptifs - ceux de Félix Deltour (1874), de Gustave Lanson (1890) et d'Antoine Albalat (1899) - comme dans les discours d'écrivains eux-mêmes - notamment d'obédience réaliste, comme ceux de Zola ${ }^{9}$, de Maupassant et de Jules Renard, rêvant à un «style pur comme l'eau est claire [...] à force de s'user, pour ainsi dire, sur les cailloux ${ }^{10}$ » (1925:711). Quelques travaux récents signalent à cet égard le moment particulier que représentent les deux dernières décennies du siècle quant à la résurgence de la valeur classique de la simplicité. Dans son Grand Dictionnaire universel, Pierre Larousse se réfère à Boileau, Rivarol et Marmontel pour définir la transparence d'un style par sa "pureté», sa "précision» et son "naturel», c'est-à-dire l'emploi des termes et des tours consacrés par la règle et l'usage, l'emploi du mot propre et le respect de la concision du discours (t. XV, 422). La transparence participe à la «grande qualité du style» (Albalat 1901: 295) et agit dans les années 1880 comme un argument

\footnotetext{
9. Zola préfère la métaphore minérale (le verre, le diamant, le cristal): depuis sa lettre à son ami Anthony Valabrègue en 1864, qui expose la fameuse théorie des «écrans", jusqu’à la «Lettre à la jeunesse» de 1896, en passant par ses deux essais de 1880 et de 1881, Le Roman expérimental et Les Romanciers naturalistes, ses écrits formulent le vœu d'une langue idéale en associant le leitmotiv de la transparence avec les valeurs classiques.

10. Extrait du Journal à la date du 8 juin 1904 .
} 
d'autorité qui rejoint largement une question proprement linguistique, celle de la défense du français comme langue nationale. En ces temps de cosmopolitisme et de métissage linguistiques ${ }^{11}$, marqués par le traumatisme militaire et politique de 1870, il s’agit aussi de rappeler que la France doit son génie à la pureté de sa belle langue.

Dans un célèbre essai qui servit de préface à Pierre et Jean, Maupassant rappelle que la transparence du beau style ne doit pas être «obscurcie» par un mot mal choisi:

La langue française, d'ailleurs, est une eau pure que les écrivains maniérés n'ont jamais pu et ne pourront jamais troubler. Chaque siècle a jeté dans ce courant limpide ses modes, ses archaïsmes prétentieux et ses préciosités, sans que rien surnage de ces tentatives inutiles, de ces efforts impuissants. La nature de cette langue est d'être claire, logique et nerveuse. Elle ne se laisse pas affaiblir, obscurcir ou corrompre (Maupassant, «Le Roman»: 715).

Pour cet écrivain réputé hostile au style maniéré des Goncourt, qu'il vise indirectement, la "pureté» de la langue française doit perdurer, par-delà les styles d'époque. Le réseau métaphorique qu'il emploie ("eau pure», "troubler», "courant limpide», "obscurcir») signale la valeur accordée à la fluidité du discours: plus le flux s'épaissit et plus il perd en clarté. Comme le montre déjà, en 1874, l'éloge d'Antoine Rivarol sur la langue française, celle-ci est associée à la clarté et à la raison, étroitement liées à la question de la propriété du lexique (1874: 100), à laquelle Maupassant consacre aussi un long développement, sous le patronage de Flaubert. Le style pur doit privilégier la «clarté» du mot propre à la "profondeur» des termes suggestifs ou à l'impropriété des synonymes en cascade (Albalat 1901: 295). Dans son Grand Dictionnaire universel du XIX siècle, Larousse évoque également avec ironie les écrivains contemporains qui préfèrent "recouvrir» leurs idées avec le "plâtras de l'amphigouri " (archaïsmes et néologismes divers) (1866-1879: t. XV). La densité du plâtre est de nouveau suscitée dans le reproche formulé par Lanson, qui déplore ces «styles pâteux» de la fin du siècle (1908: 260), ou dans cette prose "plombée» et «bitumée» que fustige l'essayiste Anatole Claveau, hermétique aux tentatives de la prose moderne (1907: 181).

La fluidité de la phrase renvoie également au caractère régulier de la syntaxe: une phrase longue doit demeurer structurée et équilibrée comme une période - dont le nombre de membres et la rondeur appellent une forme d'harmonie, à l'instar du style de Montaigne et de Bossuet (Claveau 1907 : 232-234). Quant aux modes de jonction parataxique (juxtaposition, coordination asyndétique et parataxe) et à l'accumulation de constructions détachées ou d'adjectifs, ils apparaissent souvent comme la menace d'un délitement irréversible de la syntaxe (Claveau 1907 : 175, 181). La limpidité de la phrase est ainsi comparable au développement régulier d'un flux et sa transparence évince, dans un certain discours critique, la densité obscure d'un sens à découvrir.

La complaisance des auteurs à l'égard d'images imprécises, le culte qu'ils vouent au style emphatique et abscons sont précisément ce que leur reproche Claveau, qui consacre à cette question un ouvrage de plus de trois cents pages, intitulé La Langue nouvelle ${ }^{12}$. Les productions romanesques de la fin du siècle y sont décrites par un même réseau de métaphores, celle de la «moisissure»(Claveau 1907: 3),

11. "[Les langues] voisinent trop! Les langues anciennes ont péri avec les nations qui les ont parlées; les langues modernes sont déjà victimes de cette promiscuité internationale qui les défigure en les fusionnant» (Claveau 1907: 10). Voir l'étude de Remy de Gourmont sur l'assimilation des mots étrangers (anglais, allemands) par la langue française dans l'Esthétique de la langue française, chapitres sept et huit (1899: 88-100).

12. La Langue nouvelle: Essai de critique conservatrice (1907) reprend certains articles rassemblés en 1886 dans Contre le flot (parus dans Le Figaro, Le Gaulois, Le Temps, Le Paris-Journal) et produit un ouvrage considérablement enrichi par 
du «mauvais marécage» (184), du «navire en détresse» (181), de la «fonte contondante» (183), qui connotent toutes le flottement, la dérive et l'enlisement. En exploitant un autre lieu commun de la critique, associant l'imaginaire de la pourriture à une langue faisandée, Claveau englobe dans cette «littérature déliquescente» les productions des frères Goncourt, de Zola ou de Mallarmé (Claveau 1886: 7). Pour ses détracteurs, la langue décadente est résolument une langue délitée, liquéfiée, qui déferle à l'instar d'un «flot» dévastateur. Dans son célèbre essai Dégénérescence, Max Nordau propose également le diagnostic d'un mal censé toucher les artistes de la fin du siècle en recourant à une isotopie métaphorique analogue: hantés par la sensation d'un «engloutissement» imminent (Nordau 2010: 32), qui figure la déchéance de l'humanité, les romanciers et les poètes livrent des images «nébuleusement fluides» et contraignent le lecteur à "se plonger dans la perpétuelle ébriété de phantasmes à perte de vue, sans but ni rive» (52). La métaphore de l'imagination à la dérive permet à Nordau de filer le thème de l'égarement: cette langue, qu'il qualifie de «mystique» (105), cultive l'imprécision et la suggestion, le trouble et l'opaque; pour le mélancolique décadent, le monde décrit n'est plus qu'une «masse noire ondoyante» (108), informe, épaisse et compacte.

La fluidité et la limpidité de l'eau, ainsi que leurs antonymes, la densité et l'opacité, constituent des sèmes métaphoriques récurrents en cette fin de siècle. Ce sont deux pôles, tantôt positif, tantôt négatif, sur le plan des valeurs et des normes esthétiques, qui sont éclairés par le principe diachronique de transparence linguistique. Il importe désormais de comprendre quelles peuvent être les résonances entretenues entre ces considérations stylistiques relativement générales et la nouvelle de Fleury en particulier.

\section{Esthétisation de la matière par la fantasmagorie}

Dans «Hydrargyre», deux sèmes récurrents caractérisent la Seine: la lourdeur et la densité. Le fleuve est comparé à une "nappe dense» (267-268), à "une homogène coulée de lave» (264), à un «dense métal» (269), "plus lourd que toutes les choses» (268). Par un effet d'intertextualité, la description de la Seine rappelle une page du premier roman de Huysmans, Marthe (1876), où la jeune femme, lasse et désolée, observe avec tentation "des eaux couleur de plomb» et songe à s'y précipiter (Huysmans, Marthe: 82). Chez Fleury, l'eau est également une masse substantielle, "plat[e], sans une ride» (264), suscitant la même pulsion morbide, la même force d'attraction irrépressible. Néanmoins, ce topos est réinvesti de manière à en accuser l'ambivalence. Les fantasmes que l'eau suscite évoquent d'abord un retour primitif vers une osmose maternelle et enveloppante ${ }^{13}$. La force hypnotique de l'eau s'allie au plaisir d'une incorporation et d'une absorption passive: «Un moment, stupide de terreur nocturne et d'étrangeté, il se tint raide, sans un geste; il se sentait bercé par un flot doux, caressant et souple, aux ondulations médiates d'un matelas d'eau: une invincible curiosité lui fit entr'ouvrir les paupières, il osa regarder" (264). L'immersion d'un corps raide et immobile témoigne de la compacité du flux: la liquidité devient "matelas». La suavité de l'eau ondulante ("doux, caressant et souple») ainsi que la puissance de la pulsion scopique dépossèdent le jeune amant de son pouvoir d'action; devenu "stupide», il est agi par une force suprahumaine représentée par le flot et le courant. Ce pouvoir envoûtant de la liquidité reconduit sur un autre plan (tout aussi onirique) la fascination du poète des Tableaux

de nouveaux chapitres. Le style d'époque qu'il s'attache à décrire est si répandu que Claveau dès cette période avait nommé ce premier essai de façon à souligner qu'il souhaite prendre à rebours les tendances nouvelles.

13. Le fantasme décadent d'absorption est analysé dans l'étude de Robert Ziegler Beauty Raises the Dead, notamment dans le roman Bruges-la-morte de Georges Rodenbach (2002: 38-58). 
parisiens pour l'«enivrante monotonie/Du métal, du marbre et de l'eau ( «Rêve parisien»: 101); aussi l'imaginaire peut-il se déployer par un effet subtil de résonance interne. La Seine dégage en effet chez Fleury une puissance oxymorique, à la fois salvatrice et mortifère. En témoignent les deux sèmes axiologiquement opposés de la douceur («tiède et duvetée», "frôlante», "moelleux et berceur» [270]) et de l'aspérité (couleurs «féroces», "belliqueuses» et "sanguinaires» [269]). Le fleuve est à la fois ce qui achève et ce qui soigne, ce qui scelle le mal et y remédie. D'une part, l'incantation du jeune amant blâme une puissance maléfique et destructrice, comme en témoigne cette gradation: «tu condamnes à prompte mort l'homme insolent qui te viole, tu nécroses ses os, tu dissous sa substance!» (269); d'autre part, son discours rend hommage à sa force bienfaitrice, elle seule capable de guérir du mal vénérien: "malgré toi consolateur, tu guéris les lèpres hideuses que Vénus engendra" (269). Tel le Styx de la mythologie grecque, la Seine s'avère posséder des vertus incomparables pour celui qui s'y baigne; mais sa véritable force salvatrice réside en réalité dans la mort qui libère du mal: la «terreur folle» des deux amants (272) devant l'avancée d'un bateau fatal, leurs «invincibles efforts» (272) pour remonter le fleuve à contre-courant, comme une ultime pulsion de vie, les conduisent finalement à l'épuisement et à la mort. Le bateau évoque davantage la barque de Charon faisant traverser les âmes sur le fleuve de l'Enfer que l'embarcation rédemptrice du patriarche Noé dans la Genèse.

Le fleuve est associé aux puissants effets du mercure, dont la mort par intoxication peut illustrer ce que le narrateur nomme une "terreur nocturne» (264) - terreur qui peut être celle du corps et des maladies que le sexe engendre, d'après Évanghelia Stead (2000). À cet égard, l'immersion continue des amants dans le fleuve de mercure ne procure aucun soin; elle est au contraire l'allégorie d'une complaisance autodestructrice dans la déchéance, dont l'érotisme et la sexualité ne sont qu'une modalité. Observé par le jeune savant, le corps malade de l'amante, décrit avec un vocabulaire médical spécialisé, détaillant les symptômes de la syphilis et du psoriasis, offre une beauté morbide où les contorsions et les excroissances, les pleins et les déliés dessinent sur le fleuve "un liseré de buée grasse» (270) - inscription métonymique d'une éphémère présence dont la contamination est irréversible:

Alors, il examina sa compagne que jusqu'alors il avait omis de regarder: des chapelets ganglionnaires bossuaient un peu ses aînes [sic] et son cou; au ventre et sur les seins se groupaient des papules cuivrées; quelques crins de sa chevelure se détachaient et flottaient en dérive; et dans la paume de ses mains ouvertes, le savant reconnut les chatoyantes squames du psoriasis syphilitique (269).

Cette description rappelle la putréfaction révulsante de Nana à la fin du roman de Zola (1880) et évoque la féminité mortifère et archétypale apparaissant dans le cauchemar de des Esseintes au huitième chapitre d'À Rebours (1884). Ces intertextes ont probablement influencé la représentation qu'en donne Fleury, partagée entre la sémiologie médicale et la vision fantasmagorique ${ }^{14}$. Ici encore, la précision et l'abondance du lexique scientifique ("ganglionnaires», "papules» ou "squames») contribuent précisément à l'émergence d'une figure tératologique à partir de la matérialité charnelle - la monstruosité s'inscrit dans la phusis elle-même; la nature est écart. L'observation méticuleuse d'un ensemble de symptômes pathognomoniques fait apparaître une créature fantastique dont la crinière évoque une jument, quand la perte et l'éparpillement des crins renvoient à la stérilité et la mort («se détachaient

14. L'observation érudite est liée dans le texte de Fleury à la focalisation interne ( il examina sa compagne») mais il n’est nulle part précisé dans la nouvelle que le jeune homme est un savant, un chimiste - seule la deuxième version explicite ce détail. L'originale entretient le caractère mystérieux des deux personnages anonymes et la portée allégorique du texte. 
et flottaient»); ses reliefs ("ventre» et "seins») ainsi que les excroissances répétées ("chapelets» et "papules») tracent les contours d'un paysage vallonné dont les reflets teintés par le cuivre soulignent la déréalisation onirique - explicite dans la deuxième version du texte. La jeune femme retourne ainsi, informe et difforme, à l'une des forces élémentaires de la matière. Et contrairement à ses usages médicamenteux, le mercure est ainsi dépossédé de ses vertus thérapeutiques destinées à guérir des affections syphilitiques (Larousse, t. XI : 61).

Les deux corps enlisés dans le métal liquide et fluctuant évoquent la «rêverie pétrifiante» décrite par Bachelard (1948: 195). Les personnages figés dans leur corps respectif se déplacent sans se mouvoir et, en traversant un paysage de pierres, s'acheminent inéluctablement vers leur propre perdition. Les bords de Seine prennent en effet des allures gothiques qui peuvent rappeler le Paris des eaux-fortes de Charles Meryon:

Une aube livide vert-de-grisait à l'horizon, là-bas, une bande étroite du ciel : c'était une aurore morne qu'on pressentait définitive et sans espoir de soleil à paraître; elle montrait un lointain tournant du fleuve et sur la rive droite apparaissaient, découpées sans perspective sur le fond de ternes lueurs, d'étranges monuments calcinés et fantasques, de grêles colonnades obliquement décapitées et des façades de casernes démantelées, écornées aux angles, avec des milliers de fenêtres béantes, dont les pierres grumeleuses s'effritaient, sous un déluge de corrosives pluies ${ }^{15}$ (267).

L'apparition pittoresque de ces monuments urbains ainsi détruits, comme en témoignent les participes passés ("décapitées», "démantelées», "écornées»), développe un imaginaire funèbre: ces lieux inconnus, privés de devenir, exhibent les vestiges d'une époque prestigieuse ("colonnades», "casernes») et révolue. L'aube est "morne» et "sans espoir de soleil à paraître», comme l'espace est lui-même privé de perspective. Déréalisé, le paysage est également dysphorique, évoquant ainsi la chute du "Rêve parisien» de Baudelaire qui substitue les valeurs suggestives de l'imaginaire à une vision pathétique du réel: «En rouvrant mes yeux pleins de flamme/J'ai vu l'horreur de mon taudis/[...] Et le ciel versait des ténèbres/Sur le triste monde englouti» (103). La ville trempée et pétrifiée dans sa déchéance sous le regard des deux amants présente un décor suspendu dans un hors-temps minéral et dissonant, qui réactualise l'esthétique romantique de la ruine, évoquant les tableaux de Caspar Friedrich, mais en sapant toute envolée lyrique. Les deux amants traversent ainsi «le plus sinistre des cimetières, celui où l'on n'a point de tombeau», selon Maupassant ("Sur l'eau»: 54); le deuil est éternel, la mort n’en finit pas de finir (Coquio 1993: 92).

La suite de la nouvelle minéralise encore davantage l'eau du fleuve: la pierre n'est plus le décor qui enserre le mercure liquide comme un joyau, mais elle jaillit des profondeurs de l'eau elles-mêmes. Car, parvenu au terme de son périple, le couple découvre au milieu du fleuve une "colossale obélisque» (271) sur laquelle se trouvent gravés en rouge dans la pierre noire le symbole du mercure $(\mathrm{Hg})$ ainsi que deux signes hermétiques. Le premier, qui ressemble à un esset allemand ( $(\beta)$ ou à un bêta grec $(\beta)$, est tiré de l' «Alphabet des mages» de l'alchimiste Paracelse ${ }^{16}$ et pourrait désigner la lettre « $\mathrm{H} »(\mathrm{Hg})$. Le second, apparenté à un caducée, ł̧, est le symbole astrologique de la planète Mercure; il évoque

15. L'expression «eau-forte» appelle une remarque puisqu'il s'agit précisément d'un processus d'érosion sous l'effet d'un acide. Selon le relief souhaité, l’acide (nitrique ou azotique) érode le métal (généralement le cuivre) qui est ensuite, par lithographie, appliqué au papier. On peut voir dans ces «corrosives pluies» un clin d'œil savant à «l'eau-forte» - qui mord et attaque le métal.

16. Médecin alchimiste suisse, Paracelse (1493-1541) développe une philosophie de la nature d'inspiration alchimiste qui ouvre la voie à la physiologie expérimentale et à la biochimie de la médecine moderne. L'alphabet de Paracelse, 
également, par effet de contraste ironique, les valeurs curatives du caducée médical (souvent confondu avec le bâton d'Esculape de la mythologie romaine).

Le courant a ainsi conduit les amants au «centre du grand royaume d'Hydrargyre» (271) pour mieux les anéantir en tant qu'êtres de chair; le fleuve métallique confère au minéral les fonctions symboliques de la stèle. Quant à la compacité du fleuve, elle se solde par un éparpillement effrayant lié à l'explosion ${ }^{17}$ : l'eau n'est plus une force de régénération mais le décor d'un mysticisme sans dieu ${ }^{18}$. Ces nouveaux Adam et Ève, qui commettent le péché de la chair («ils se donnèrent l’un à l'autre» [270]), ne parviennent pas à régénérer l'humanité; au contraire, l'imaginaire nourrit une relation étroite entre dégénérescence des corps et plaisir vénérien. À cet égard, la référence à la syphilis - topique dans les années 1880 - souligne une "méfiance de la chair ", la maladie apparaissant comme un "legs maudit» dont l'origine obscure "laïcise» le péché originel et le traduit en termes biologiques (Cabanès 1996: 89-92). Le sens impénétrable, auréolé du prestige du sacré antique ou primitif, se laisse approcher à l'instant même où il échappe aux personnages. Dans une eau lourde et profonde, les deux rescapés sans résistance redeviennent des mortels en sursis: "un choc allait suffire à l'effroyable éparpillement de tout par le mélange détonant» (272). La métallisation de l'eau, toxique et nocive, ainsi que sa minéralisation fatale sont ainsi lues comme une allégorie de la fin du monde qui hante les consciences décadentes de la fin du siècle. À l'éclatement des corps, à leur déliaison future, s'adjoint leur engloutissement imminent - lieux communs d'une poétique notamment étudiés par Max Nordau (2010: 32) et Vladimir Jankélévitch $\left(1950: 349^{19}\right)$.

La longue traversée des amants dans le fleuve de mercure, à la fois liquide et minéral, s'appuie ainsi sur une esthétique de la déliaison, confortée par le motif de l'effritement des pierres qui bordent la Seine. Il convient d'observer la façon dont cette esthétique se manifeste par des choix stylistiques spécifiques.

\section{Matière liquide et style diffus}

Sur le plan syntaxique, la nouvelle de Fleury recourt fréquemment à la juxtaposition asyndétique de syntagmes ${ }^{20}$ et à l'accumulation de constructions syntaxiques effaçables (non essentielles du point de vue grammatical). À titre d'exemple, évoquons la fréquence des appositions de participes passés employés comme adjectifs ( démantelées, découpées sans perspective [...], écornées aux angles»), ainsi que celle des compléments circonstanciels, dits accessoires ("avec des milliers de fenêtres béantes [...], sous un déluge de corrosives pluies» [267]). Ces appositions et ces circonstants sont, sur le plan de la

appelé également "Alphabet des mages» ou des "anges", est destiné à graver le nom des anges sur les talismans censés guérir ceux qui les portent.

17. Au Xix ${ }^{\mathrm{e}}$ siècle, le principal agent explosif employé dans les armes à feu est précisément le fulminate de mercure.

18. "Quand il n'y a plus de religion, le mysticisme court les rues» (Charbonnel 1897: 5).

19. Dans le premier tome de Dégénérescence, Nordau décrit la "disposition d'esprit crépusculaire» (2010: 75) caractéristique de la fin du XIX ${ }^{\mathrm{e}}$ siècle en recourant à la métaphore de l'engloutissement: cette dégénération pathologique de la culture occidentale, dont la littérature décadente n'est qu'un épiphénomène, s'exprime par l'aboulie, l'adynamie, des facultés étiolées; «l'homme du crépuscule» (50), incapable d'agir, se laisse délibérément «engloutir» (32), «sombrer", "plonger» dans ses névroses (52). Jankélévitch insiste quant à lui sur le thème de la disjonction (1950: 349355) : comme poétique de l'excès, la décadence consiste pour Jankélévitch en une distorsion des formes physiques aussi bien que stylistiques, en rapprochant tératogonie et «expressionnisme» (351).

20. L'asyndète se caractérise par l'absence de termes coordonnants entre un groupe de mots ou entre plusieurs phrases (par exemple: bon gré, mal gré). 
syntaxe, des syntagmes mobiles et suppressibles dont la valeur essentiellement descriptive conforte, sur le plan stylistique, la poétique de la déliaison.

La récurrence des phrases longues participe également d'un style «diffus» (Marmontel 1787: 409-411) dont le discours critique des années 1880 regrette le relâchement et le défaut de concision (Lanson 1890: 230-232). Cette «faiblesse» syntaxique est censée être compensée par les recherches relatives au lexique (Marmontel 1787: 386). Or, la précision du vocabulaire scientifique (chimique et médical) dans la nouvelle de Fleury rend compte de son souci de la propriété lexicale. En témoigne ce passage où, une fois le fleuve ralenti, les deux amants se découvrent à la campagne: "Par-delà s'étalait le désert, d'interminables plages pulvérulentes, ocreuses de turbith minéral, coupées de veines verdâtres, et que mamelonnaient par places de basses dunes arrondies, faites d'éthiops calcaire à la couleur grise» (267; nous soulignons). Ainsi décrit par le biais d'un vocabulaire technique (en italique) ou rare («mamelonner»), le «désert» des bords de Seine inverse un lieu commun: le locus amoenus devient une mosaïque étrange de formes irrégulières et de couleurs glauques. Le laid et le mortifère sont alors transfigurés par le travail esthétisant de la prose.

La nouvelle est par ailleurs ponctuée de néologismes (comme «flabellaire», néologisme adjectival dérivé de l'adjectif «flabellé» [264]); l'emploi de génitifs bibliques permet également de transformer une caractéristique en substance en privilégiant un désadjectival («la vélocité de leur course» [266], "l'intimité de son cour» [266], "la soudaineté d'un jeu de glaces» [267; nous soulignons]); enfin, quelques cas de déplacements sémantiques, comme les hypallages ("creuset nomade» [265], "aurore morne» [267], "amoureux sourire» [270], «lenteur féroce» [272], «barque fatale» [272; nous soulignons]), et des cas d'impropriétés grammaticales, comme l'énallage (un "lyrisme, affolé d'étrange» [269; nous soulignons]), participent du projet d'esthétisation de la prose, si peu compris par la critique conservatrice de la fin du siècle. D'autres faits de style lexico-syntaxiques y contribuent, comme l'antéposition de l'adjectif, la fréquence des adverbes et celle des participes présents, censés "plomber» le style (Claveau 1907: 103). Tout se passe comme si la nouvelle de Fleury faisait se rejoindre deux types d'imagination distincts, celui de la matière linguistique et celui du motif littéraire et scientifique.

De fait, ce fleuve compact, dense, obscur, qui emporte les deux personnages vers un sens suggestif et ouvert entre clairement en résonance avec les problématiques stylistiques de l'époque sur la transparence, la fluidité et la clarté de la belle langue. Ce fleuve tout en "coulées», en "nappes» et en "plaques» (264), c'est-à-dire en parties compactes mais détachables, est l'une des manifestations du délitement syntaxique dont s'inquiète la critique à la fin de siècle, et qui sont notamment observables dans le rythme cahotant des syntagmes détachés par les virgules. Là encore, c'est la fréquence des compléments circonstanciels et celle des groupes adjectivaux apposés qui soulignent l'expolition, la description par touches successives du délitement d'une surface miroitante:

Tout au ras de sa vue, ainsi qu'un lit géant, le fleuve en nappe indéfinie s'étalait, plat, sans une ride, plus poli qu'un miroir, glissant d'une homogène coulée de lave; des plaques, çà et là, semblaient saupoudrées de poussière, et tout le reste, d'un luisant bleu de lame neuve, répercutait avec une pureté froide et la plus absolue netteté, aux bords, le pan noir de quais démesurés, au milieu, le disque lunaire, tout rond, non pourvu du panache flabellaire qu'il laisse traîner sur les eaux, si précisément redit, si brillant, qu'à le regarder il louchait, le voyait double; autour, myriadaient les gouttelettes diamantées des étoiles (264; nous soulignons).

Le miroitement du métal liquide qui reflète les quais noirs des bords de Seine et l'obscurité scintillante de la nuit ne donnent plus à voir en transparence la claire profondeur de ses eaux; la préciosité du 
lexique et l'expressivité des métaphores (la «lame», le diamant) invitent le lecteur à un cheminement herméneutique. Cette prose poétique «tire l'œil» du lecteur (Claveau 1907: 195) vers la matérialité même du texte, la scénographie du style, les foyers lumineux du lexique - qu'il s'agisse d'un néologisme ( «myriader», néologisme verbal par affixation dérivationnelle sur la base du substantif «myriade») ou d'un adjectif substantivé ( un luisant»).

Sans promouvoir un formalisme pur, sans jouer contre les significations, cette prose à effets suppose un mouvement de contorsion autotélique, comme si, in fine, la transparence se changeait en miroir.

Ainsi, la nouvelle revêt une dimension réflexive qui fait partie intégrante de l'ambition littéraire de son auteur. La puissance ambivalente de la Seine, qui donne cours à une rêverie métallique sur la matière mobile et changeante, ne laisse rien paraître de cristallin ni d'univoque. Ce fleuve opaque et dense souligne au contraire la plasticité du style littéraire lui-même, celle d'une "prose moderne » saluée par Paul Bourget en 1883 (1993: 335), mais comparée aux «marécages» par ses détracteurs (Claveau 1907 : 184). Le mysticisme des motifs symboliques et leur syncrétisme, la représentation esthétisée des corps morbides, la fascination qu'exerce la matière élémentaire (liquide, minérale, biologique) ainsi que l'intertexte baudelairien procèdent résolument de la poétique décadente de la fin du siècle. À ce titre, «Hydrargyre» constitue une tentative éloquente: celle de représenter, par la fiction narrative, la fin d'une utopie linguistique, et de consacrer, par le recours même à la matière linguistique savante, la faillite du rêve d'une langue transparente, "miroir sans buée d'une connaissance» (Foucault 1966: 309).

\section{Références bibliographiques}

Albalat, A. 1899. L'Art d'écrire enseigné en vingt leçons. Paris: Armand Colin et Cie.

Albalat, A. 1901. La Formation du style par l'assimilation des auteurs. Paris: Armand Colin.

Bachelard, G. 1942. L'Eau et les Rêves: essai sur l'imagination de la matière. Paris: Le Livre de Poche.

Bachelard, G. 2004. La Terre et rêveries de la volonté: essai sur l'imagination de la matière. Paris: Corti. [1948].

Baldick, R. 1958. La Vie de J.-K. Huysmans. Traduit par M. Thomas. Paris: Denoël.

Barre, A. 1911. Le Symbolisme. Paris: Jouve et Cie éditeurs.

Baudelaire, Ch. 1975. "Rêve parisien». Dans Les Fleurs du Mal. Dans Euvres complètes, texte établi, présenté et annoté par C. Pichois. Paris: Gallimard: t. I, 101-103. [1860].

Bouhours, D. 1673. Les Entretiens d'Ariste et d'Eugène. Paris: Sébastien Mabre-Cramoisy. [1671].

Bourget, P. 1993. Essais de psychologie contemporaine: études littéraires. Édition préparée par A. Guyaux. Paris: Gallimard. [1883].

Bury, M. 1990. «Les écrivains décadents de la fin du XIx siècle et le sacré», Bulletin de l'Association Guillaume Budé 1 (3) : 308-316.

Bury, M. 2004. La Nostalgie du simple: essai sur les représentations de la simplicité dans le discours critique au $X I X^{e}$ siècle. Paris: Honoré Champion.

Brogniez, L. 2007. «La ville et ses doubles: Les paysages urbains de Francis Poictevin». Dans Les Villes du symbolisme, sous la direction de M. Quaghebeur et M.-F. Renard. Bruxelles: Peter Lang: 183-206.

Cabanès, J.-L. 1996. «Invention(s) de la syphilis». Romantisme 94: 89-109.

Charbonnel, V. 1897. Les Mystiques dans la littérature présente. Paris: Mercure de France.

Citti, P. 1987. Contre la Décadence: histoire de l'imagination dans le roman, 1890-1914. Paris: Presses universitaires de France. 
Claveau, A. 1886. Contre le Flot. Paris: Paul Ollendorff.

Claveau, A. 1907. La Langue nouvelle: essai de critique conservatrice. Paris: Librairies-Imprimeries réunies.

Coquio, C., 1993. "La “'baudelairité” décadente: un modèle spectral». Romantisme 82: 91-107.

Deltour, F. 1878. Principes de composition et de style. Paris: Delagrave. [1874].

Emery, E. et J. Forrest, dir. 2017. "Matter Pools: Fluidity and Containment in French Decadent Literature.

Essays in Honour of Robert E. Ziegler». Dix-neuf 21 : 93-103.

Fleury, M. 1887. «Hydrargyre». Revue indépendante 7: 263-273.

Fleury, M. 1891. Amours de savants. Paris: Charpentier.

Fleury, M. 1896. «Au jour le jour: le livre sur Émile Zola». Le Figaro 6 novembre: 1.

Fleury, M. 1897. Introduction à la médecine de l'esprit. Paris: F. Alcan.

Foucault, M. 1966. Les Mots et les Choses: une archéologie des sciences humaines. Paris: Gallimard.

Gourmont, R. 1899. Esthétique de la langue française: la déformation, la métaphore, le cliché, le vers libre, le vers populaire. Paris: Société du Mercure de France.

Huysmans, J.-K. 1975. Marthe: histoire d'une fille. Dans Les Sæeurs Vatard. Préface d'Hubert Juin. Paris: Union générale d'éditions. [1876].

Jankélévitch, V. 1950. «La décadence». Revue de métaphysique et de morale. CNRS, 55 (4) octobre-décembre: 337-369.

Joly-Segalen, A. et G. Germain. 1964. «Lettres inédites de Victor Segalen. L'entrée de Segalen au Mercure de France. Le Prix Goncourt $1907 »$. Annales de Bretagne 71 (3): 429-443.

Lanson, G. 1890. Conseils sur l'art d'écrire: principes de composition et de style à l'usage des élèves des lycées et collèges et de l'enseignement primaire supérieur. Paris: Hachette.

Lanson, G. 1908. L'Art de la prose. Paris: Librairie des Annales politiques et littéraires.

Larousse, P. 1982. Grand Dictionnaire universel du XIX siècle. Paris: Administration du Grand Dictionnaire universel; Genève: Slatkine: t. XI, entrée «Mercure», t. XV, entrée "Transparence». [1866-1879].

Marmontel, J.-F. 2005. Eléments de littérature. Édition préparée par S. Le Ménahèze. Paris: Desjonquères. [1787].

Maupassant, G. 1974. «Sur l'eau». Dans Contes et Nouvelles, édition préparée par L. Forestier. Paris: Gallimard: t. I., 51-57. [1876].

Maupassant, G. 1987. «Le Roman». Dans Romans, édition préparée par L. Forestier. Paris: Gallimard: 703715. [1887].

Mitterand, H. 1985. "De l'écriture au style décadent». Dans Histoire de la langue française 1880-1914, sous la direction de G. Antoine et R. Martin. Paris: Éditions du Centre national de la recherche scientifique: 467-477.

Nordau, M. 2010. Dégénérescence. Traduit par A. Dietrich. Lausanne: L’Âge d'homme. [1892].

Paulhan, F. 1891. Le nouveau Mysticisme. Paris: F. Alcan.

Pichois, C. 1975. «Notice, notes et variantes». Dans Ch. Baudelaire, Euvres complètes, texte établi, présenté et annoté par C. Pichois. Paris: Gallimard: t. I, 787-1224.

Philippe, G. 2002. Sujet, verbe, complément: le moment grammatical de la littérature française, 1890-1940. Paris: Gallimard.

Philippe, G. 2013. Le Rêve du style parfait. Paris: Presses universitaires de France.

Philippe, G. et J. Piat, dir. 2009. La Langue littéraire: une histoire de la prose en France de Gustave Flaubert à Claude Simon. Paris: Fayard.

Pierrot, J. 1977. L'Imaginaire décadent (1880-1890). Paris: Presses universitaires de France; Rouen: Publications des Universités de Rouen et du Havre.

Renard, J. 1990. Journal (1887-1910). Édition préparée par H. Bouillier. Paris: Robert Laffont. [1925].

Richard, J.-P. 1984. "Quais de Seine». Dans Pages Paysages: Microlectures II. Paris: Seuil: 101-108.

Rivarol, A. 2013. Discours sur l'universalité de la langue française, précédé de La Langue humaine par G. Dessons. Paris: Manucius. [1874]. 
Rodenbach, G. 2002. Bruges-la-morte. Bruxelles: Labor. [1892]

Segalen, V. 1902. L’Observation médicale chez les écrivains naturalistes. Paris: Y. Cadoret.

Schuré, É. 1983. Les Grands Initiés. Paris : Librairie académique Perrin. [1889].

Stead, É. 2000. "Odilon Redon dans les textes belges et français de la décadence: Les images invisibles", Textyles 17-18:55-72.

Stead, É. 2004. Le Monstre, le Singe et le Foetus: Tératogonie et décadence. Genève: Droz.

Ziegler, R. 2002. Beauty Raises the Dead: Literature and Loss in the Fin de Siècle. Newark: University of Delaware Press. 